\title{
Prophylactic antibiotics with operative vaginal delivery: a cost-utility analysis based on the ANODE trial.
}

\author{
Ritvij Singh ${ }^{1}$, Nivaran Aojula ${ }^{2}$, and Tadeusz Ciecierski-Holmes ${ }^{3}$ \\ ${ }^{1}$ Imperial College London \\ ${ }^{2}$ Imperial College London Department of Medicine \\ ${ }^{3}$ Independent
}

July 6, 2020

\begin{abstract}
Objective To perform a cost utility analysis of prophylactic antibiotics compared with a placebo in preventing maternal infection after operative vaginal birth. Design Economic analysis based on the findings of the ANODE trial, a multi-centre randomised placebo-controlled trial. Setting Twenty-seven hospital obstetric units across the UK. Population or Sample Women who underwent operative vaginal delivery after at least 36 weeks of gestation. Methods A cost utility analysis from the perspective of the National Health Service was performed. To inform the decision analysis, a decision tree was constructed to calculate an incremental cost effectiveness ratio (ICER). Two one-way sensitivity analyses were conducted. Main Outcome Measures Difference in quality of life and cost between patients given the antibiotics and the placebo. Quality of life scores were reported EQ-5D-5L measurements from the ANODE trial. Results The ANODE trial reported a statistically significant reduction in infection rates with the co-amoxiclav intervention, this was found to yield an improvement of 0.017 QALYs over the placebo1. The cost to the NHS of the intervention was also lower at $£ 120$ over 6 weeks compared to the placebo's £194. This gives an ICER of -£4403.14/QALY. Sensitivity analysis confirmed that the intervention was dominant. Conclusions A prophylactic dose of co-amoxiclav following operative vaginal delivery is a cost-effective intervention, offering improved QoL whilst also reducing overall costs over a 6 -week time period. Tweetable abstract Prophylactic antibiotics are a cost-effective way to reduce maternal infection after operative vaginal delivery.
\end{abstract}

\section{Introduction}

Maternal infection following delivery of a foetus can result in life-threatening complications such as sepsis. This accounts for $7 \%$ of maternal deaths in the UK post-partum ${ }^{1}$. Treatment of serious complications such as sepsis places a large financial burden on the NHS, with all sepsis treatment reported to cost the NHS £2.5 billion annually ${ }^{2}$.

Key determinants of maternal sepsis include interventional deliveries such as caesarean section and operative vaginal delivery, with infection occurring in approximately $20 \%$ and $16 \%$ of cases respectively ${ }^{1}$. Caesarean section involves an abdominal incision to deliver the foetus. Operative vaginal delivery - occurring in approximately $13 \%$ of pregnancies - involves the delivery of the foetus through the vagina using forceps or ventouse (vacuum $)^{1}$. For the former, antibiotic prophylaxis is recommended as the gold-standard practice by NICE (National Institute of Clinical Excellence) ${ }^{3}$, with evidence reporting up to a $70 \%$ decrease in wound infection and severe maternal infection ${ }^{1}$. On the other hand, recommendation for prophylactic antibiotics to be given for operative vaginal delivery was only given in 2020 by the Royal College of Obstetricians and Gynaecologists $^{4}$ in response to the results of the ANODE trial.

This paper is an economic evaluation based on data obtained from the ANODE trial ${ }^{1}$. The objective of the evaluation is to compare the costs and benefits yielded by a single dose of co-amoxiclav with that of a 
placebo following operative vaginal delivery over a 6 -week time horizon. This will inform whether in addition to being an efficacious practice, it is a cost effective one too.

\section{Methods}

\section{Study design and participants}

The ANODE trial is a is a multi-centre, double-blind, randomised, placebo-controlled trial that was run between March 2013 and June 2018 ${ }^{1}$. The inclusion criteria, exclusion criteria and ethics approval for the ANODE trial can be found in their appendix ${ }^{1}$. This study is a cost-utility analysis based on the primary data it reported and as such there was no patient or public involvement. In addition, this study received no external funding.

\section{Choice of analysis and perspective}

A cost-utility analysis using a decision tree was conducted in this economic evaluation. Maternal quality of life, represented by the mean EQ-5D-5L scores in the ANODE trial, was used as a measure of benefit and cost data was recorded in monetary units (PSs). The time horizon chosen is 6-weeks, this is because the majority of maternal infections are likely to occur within the first 6-7 days following delivery and resolve within the 6 -week time period ${ }^{5}$. To enable comparison with the NICE 'cost-effective' threshold, the outcomes will be expressed as cost per QALY. In calculating the QALYs an assumption will be made that the quality of life (QoL) of patients during the 6-week period is constant and will be scaled to one year.

The choice of perspective used is the UK's National Health Service (NHS). This is because as a publicly funded healthcare system it has a pressure to deliver cost effective care. Also, the Infection resulting from operative vaginal delivery impacts both primary and secondary care services which are both provided by the NHS and so provides a holistic view of the total economic costs of the intervention.

\section{Intervention}

Women who underwent forceps or vacuum delivery after gestation periods of at least 36 weeks were randomised to receive a single prophylactic dose of IV co-amoxiclav provided there were no contraindications. A placebo ( $0.9 \%$ saline) was chosen as the comparator in this economic evaluation as it was in the ANODE trial ${ }^{1}$. This is because current WHO recommendations state that no antibiotic prophylaxis should be provided following operative vaginal delivery ${ }^{6}$.

\section{Decision tree}

Figure 1 shows the decision tree. The decision node splits the tree into two arms:

1. Arm 1: the new intervention of providing intravenous co-amoxiclav.

2. Arm 2 : the current standard practice of offering no antibiotic prophylaxis after operative vaginal delivery; this is represented by the data for the placebo cohort of the ANODE trial.

The assumptions made in constructing the disease pathway reflected in the tree were necessary to clean the data and are as follows ${ }^{7}$ :

- A patient with no perineal pain would not experience infection

- Endometritis is mutually exclusive to perineal wound infection

- All patients with perineal wound infections would progress to develop perineal wound breakdown.

The unit cost of resources are outlined in Table $1^{8-15}$. An explanation of how results from the trial were used to estimate resource use and calculate final terminal node costs can be found in Appendix S1.

The data provided in the ANODE trial posed two issues with regards to modelling.

1. The ANODE trial reported outcomes as independent events ${ }^{1}$.

2. Not all patients that reported primary outcomes had their secondary outcomes measured. This was 3420 patients and 2593 patients respectively ${ }^{1}$. 
Solution to Problem 1: Constructing the decision tree with the numbers reported in the

ANODE trial would imply that the outcomes were independent events ${ }^{1}$. However, the

likelihood of a clinical outcome such as wound breakdown occurring is affected by other

outcomes such as whether the patient experienced perineal pain. For this reason, using medical literature and an understanding of the healthcare delivery process associated with postpartum infection, conditional probabilities were calculated.

Solution to Problem 2: The decision tree has mutually exclusive nodes that are combinations of primary and secondary outcomes. The nominal reported numbers of patients experiencing secondary outcomes could not be used to calculate conditional probabilities as they would be an underrepresentation. For this reason, the number of individuals experiencing secondary outcomes was standardised by scaling the number up to represent the number of expected outcomes for a cohort of 3420 participants.

\section{Outcomes}

Only clinical outcomes were used as endpoints in the analysis, as shown in Figure 1. The reasons why certain secondary outcomes were not explicitly included in the decision tree are shown in Appendix S2. While these outcomes were not assigned individual nodes, the use of NHS guidelines (Appendix S3) allowed for the cost of these outcomes to be grouped and used in the cost of treatment complications included in the decision tree.

The data on outcomes was collected in two ways in the ANODE trial. Firstly, the number of people with one of the primary outcomes of suspected maternal infection was recorded 6 weeks after delivery through telephone interviews and hospital records. The secondary outcomes were collected based on a questionnaire also completed at the same time and included their Mean EQ-5D-5L values ${ }^{1}$.

The EQ-5D-5L score ranges from 0 to 1 , with a higher score indicating greater quality of life. The ANODE trial reported a mean EQ-5D-5L score of 0.935 and 0.927 for the co-amoxiclav and placebo groups respectively ${ }^{1}$. However, within these groups there was variation that needed to be considered such as those patients that experienced sepsis or no infection at all. To account for this, an EQ-5D-5L score for sepsis of 0.848 was acquired from the literature ${ }^{16}$. All other endpoints of the decision tree involved forms of localised infection or systemic infection not resulting in sepsis and were accordingly attributed the mean EQ-5D-5L value reported in the ANODE trial ${ }^{1}$. Due to the short 6-week time horizon, no discounting of benefit data was required ${ }^{17}$.

\section{Unit costs}

Cost of intervention: The intervention is a single dose of IV co-amoxiclav, given post-delivery. Since coamoxiclav is already widely used within the NHS, its cost could be reliably estimated from the British National Formulary (BNF). The ANODE trial reports an average NHS indicative cost for the 3 main providers of co-amoxiclav powder calculated using the BNF $2017^{8}$, totalling PS2.30. For this economic evaluation, the same methodology was employed but with updated costs from the BNF $2020^{13}$, yielding an average price of PS2.26. A placebo of $0.9 \%$ saline was the comparator and just as in the ANODE trial this was attributed no cost.

Cost of complications: In order for the cost of complications to be accurate, the typical patient journey and the likelihood of using certain NHS resources over that journey needed to be considered. This was informed by an NHS flowchart obtained from the Royal Berkshire Hospital NHS Foundation Trust guidelines on postpartum infection (Appendix S3). This highlighted the role of home visits by midwives as the first healthcare point of contact for mothers with suspected postpartum complications and this was consequently included in all cost estimates. Literature searches on standard treatments for each specific complication informed the cost at each terminal node. In certain cases, assumptions were made to estimate the costs of specific complications. A cost for systemic infection but without subsequent development of sepsis was not obtainable; therefore, the treatment cost associated with sepsis was utilised for all outcomes of systemic 
infection. This is justified as irrespective of the development of sepsis, systemic infections will necessitate similar treatment costs, as reflected in the National Tariff ${ }^{17}$. Other assumptions included that patients were only re-admitted into hospital for systemic infection, endometritis and wound breakdown and that all patients with "other or confirmed suspected infections" were treated in the community.

Discounting : To ensure all costs were accurate, sources of cost data used were UK-based and from the year of the trial (March 2016-) or newer. Moreover, all but one of the costs were taken directly from NHS reference costs, the British National Formulary (BNF) or government reports. Historical costs were discounted at a rate of $3.5 \%$ in line with NICE guidelines ${ }^{18}$, converting all costs into the year 2020.

Results

Cost-utility analysis

The decision tree shows the expected cost for the 6-week period as PS120.07 for the co-amoxiclav arm and PS193.96 for the placebo arm. Moreover, the expected utility of those in the co-amoxiclav arm was 0.986, compared to 0.969 in the placebo cohort.

$$
I C E R=\frac{\operatorname{COST}(\text { Antibiotic })-C O S T(\text { Placebo })}{\text { QoL }(\text { Antibiotic })-Q o L(\text { Placebo })}=\frac{£ 120.07-£ 193.96}{0.986-0.969}=-£ 4403.14 \text { per QoL }
$$

The ICER calculated was -£4404.14 per QOL. An assumption can be made that patients' QOL will remain stable over the course of 1 year, this gives an ICER of -£4404.14/QALY. As shown in Figure 2, the results of the analysis indicate that the activity (co-amoxiclav prophylaxis) dominates the current practice of providing no antibiotic prophylaxis (placebo). It provides greater benefit at a lower cost; thus, making it a cost-effective intervention, considerably lower than the cost-effectiveness threshold of $£ 30,000 /$ QALY.

Net Monetary Benefit (NMB) and Net Health Benefit (NHB): calculation of the NMB and NHB provides a means of expressing the ICER in terms of a monetary or health unit, catering for simpler interpretation. Using the NICE threshold of $£ 30,000 /$ QALY, the NMB is $£ 577.34$ and the NHB is 0.019 QALYs. As these values are greater than 0 , the intervention is deemed to be cost-effective, subject to sensitivity analysis. The NHB of 0.019 QALYs indicates that co-amoxiclav results in a gain of 0.019 QALYs, where each QALY is valued at $£ 30,000$.

Sensitivity analysis

This section explores three sensitivity analyses. Two one-way sensitivity analyses were conducted in order to test the uncertainty of the modelling given our assumptions. A further probabilistic sensitivity analysis was performed to test the model given uncertainty in the true population parameters in the available sample.

Preliminary analysis demonstrated that the cost of perineal wound breakdown treatment impacted the ICER most greatly. In the given model, the estimate of this cost incorporated maternal readmission to hospital. The proportion of cases that were referred to hospital was based on the ANODE trial's maternal readmission rate. However, wound breakdown can also be treated in the community ${ }^{1}$. Therefore, sensitivity analysis was conducted based on community treatment costs. Assuming QOL remains consistent over a period of a year, this analysis yielded a new ICER of $-£ 3635.35 /$ QALY, indicating cost savings of $£ 3635.35$ for an extra QALY gained.

A second sensitivity analysis was conducted on the utility parameter for patients that had perineal pain but no infection. In the original model, the utility score assigned to this patient group was 1 . However due to the short time-period of 6 -weeks, it is possible that this group of patients also experienced a lower QoL. Therefore, sensitivity analysis was conducted by attributing this patient group the mean utility values provided in the trial of 0.935 for the co-amoxiclav cohort and 0.927 for the placebo cohort. This yielded an ICER of -£7203.33/QALY, corresponding to cost savings of $£ 7203.33$ per extra QALY gained. 
Under both sensitivity analyses, co-amoxiclav continued to be a dominant activity (Figure 2) and all ICERs reported were negative. Overall, the analysis showed that the intervention became less cost effective when the cost of perineal wound breakdown varied but more cost effective when the utility of patients with pain but no infection was reduced.

A third sensitivity analysis was conducted testing uncertainty of the true population parameters of the model, given the sample provided by the ANODE trial. Figure 3 demonstrates the results of a Monte Carlo simulation, run 10,000 times, using the sample probabilities presented in the decision-tree in Figure 1. The redellipse captures the $95 \%$ confidence interval of the simulation results, where the $95 \%$ confidence interval for the intervention ICER has lower-bound of - £6701.33/QALY and an upper-bound ICER of £1874.36/QALY. Therefore, the intervention remains cost-saving and QALY improving with a high-degree of significance when presented with parameter uncertainty, and the intervention is far below the NICE cost per QALY thresholds.

\section{Discussion}

\section{Strengths and limitations}

Given that the data came from a range of regions in the $\mathrm{UK}^{1}$, the results of this economic evaluation are generalisable across the NHS. Considerable differences exist in infrastructure and resource availability between high and low-income countries and so further research is required before these results can be generalised internationally.

The main limitation of this economic evaluation was the need to make assumptions regarding the patient journey to construct the decision tree model. In this process some branches may have been omitted, notably that of patients with no pain but relevant perineum related complications.

\section{Comparison with the literature}

Although this study marks the first comprehensive economic evaluation for this intervention, the findings of it align with those reported in an abstract by Owens ${ }^{19}$. This US study also found co-amoxiclav prophylaxis to be a dominant strategy - even following sensitivity analysis ${ }^{19}$. Although there are differences in demographics and cost of treatments between the two countries, the alignment of findings further reiterates the prospective role of co-amoxiclav in the prevention of maternal infection.

\section{Conclusions}

This economic evaluation concludes that the provision of intravenous co-amoxiclav following operative vaginal delivery is a cost-effective intervention, offering improved QOL whilst also reducing overall costs over a 6 -week time period. Prior to this study, existing literature regarding the cost-effectiveness of this intervention was sparse; consequently, this study significantly adds to the literature as the first UK economic evaluation to be conducted for this intervention. Importantly, we recommend an update to current NICE guidelines, so that administration of co-amoxiclav following operative vaginal delivery is viewed as the gold-standard practice. Further research should focus on identifying and assessing the cost and benefit of alternative antibiotics against co-amoxiclav

\section{Disclosure of interests}

The authors have no interests to disclose

\section{Funding}

The authors received no specific funding for this work.

Details of ethics approval

No ethical approval was necessary because all data was collected from the public domain.

Contribution to authorship 
RVS and NA wrote the first draft and contributed to the analysis of the data. TCH performed the probabilistic sensitivity analysis. The final version of the manuscript was approved for publication by RVS, NA and TCH

\section{Acknowledgments}

We thank our supervisor Laure de Preux at Imperial College London as well as the participants and researchers on the ANODE trial

\section{References}

1. Knight M, Chiocchia V, Partlett C, Rivero-Arias O, Hua X, Hinshaw K. Prophylactic antibiotics in the prevention of infection after operative vaginal delivery (ANODE): a multicentre randomised controlled trial. The Lancet. 2019;393(10189):2395-2403.

2. Leading Change, Adding Value Team. Improvement in early detection and prompt treatment of sepsis across Weston Area Health NHS Trust. The Atlas of Shared Learning, NHS England [Internet]. 2018 [cited 2020 February 15]. Available from:https://www.england.nhs.uk/atlas_case_study/improvementin-early-detection-and-prompt-treatment-of-sepsis-across-weston-area-health-nhs-trust/.

3. National Institute of Health and Care Excellence. Caesarean Section. NICE Guidance [Internet]. 2011 [cited 2020 February 15]. Available from:https://www.nice.org.uk/guidance/cg132/documents/caesarean-section-update-nice-guideline2.

4. Murphy DJ, Strachan BK, Bahl R, on behalf of the Royal College of Obstetricians Gynaecologists. Assisted Vaginal Birth. BJOG 2020; https://doi.org/10.1111/1471-0528.16092. 00: 1- 43.

5. Mohamed-Ahmed O, Hinshaw K, Knight M. Operative vaginal delivery and post-partum infection. Best Practice \& Research Clinical Obstetrics \& Gynaecology. 2019;56:93-106.

6. World Health Organisation. WHO recommendation against routine antibiotic prophylaxis for women undergoing operative vaginal birth [Internet]. Geneva: The WHO Reproductive Health Library; 2015 [cited 2020 February 15]. Available from: https://extranet.who.int/rhl/topics/preconceptionpregnancy-childbirth-and-postpartum-care/who-recommendation-against-routine-antibioticprophylaxis-women-undergoing-operative-vaginal-birth.

7. KEMH Obsetrics \& Gynaecology. Postpartum infections: treatment and management [Internet]. 2017 [cited 2020 February 2020]. Available from: https://kemh.health.wa.gov.au/ /media/Files/Hospitals/WNHS/For health professionals/Clinical guidelines/OG/WNHS.OG.InfectionPostnatalInfectionTreatment.pdf.

8. Joint Formulary Committee. British National Formulary (online) [Internet]. London: BMH Group and Pharmaceutical Press; 2017 [cited 2020 February 2020]. Available from: http://www.medicinescomplete.com.

9. Curtis LA, Burns A. Unit Costs of Health and Social Care 2019. Canterbury: Personal Social Service Research Unit, University of Kent; 2019.

10. Reference costs 2017/2018: highlights, analysis and introduction to the data [Internet]. London: NHS Improvement. 2018 - [cited when?]. Available from: https://improvement.nhs.uk/resources/referencecosts/

11. Guest J, Fuller G, Vowden P. Costs and outcomes in evaluating management of unhealed surgical wounds in the community in clinical practice in the UK: a cohort study. BMJ Open. 2018;8(12):e022591.

12. Harding, D. Postpartum Endometritis. Patient.info [Internet]. 2017 [cited 2020 February 15]. Available from: https://patient.info/doctor/postpartum-endometritis-pro.

13. Joint Formulary Committee. British National Formulary (online) [Internet]. London: BMH Group and Pharmaceutical Press; 2020 [cited 2020 February 2020]. Available from: http://www.medicinescomplete.com.

14. Drug Tariff. NHS Prescription Services [Internet]. NHS Business Service Authority. c2020 [cited 2020 February 15]. Available from: https://www.nhsbsa.nhs.uk/pharmacies-gp-practices-andappliance-contractors/drug-tariff.

15. Hex N, Retzler J, Bartlett C, and Arber M. The Cost of Sepsis Care in the UK: Final Report. York Health Economics Consortium. 2017 [cited 2020 February 15]. Available from: 
http://allcatsrgrey.org.uk/wp/wpfb-file/yhec-sepsis-report-17-02-17-final-pdf/.

16. He X, Liao X, Xie Z, Han L, Yang X, Kang Y. Pulmonary Infection Is an Independent Risk Factor for Long-Term Mortality and Quality of Life for Sepsis Patients. BioMed Research International. 2016:1-10.

17. 2019/20 National tariff payment system. NHS Improvement [Internet]. 2019 [cited 2020 June 21]. Available from: https://improvement.nhs.uk/resources/national-tariff/\#h2-tariff-documents.

18. NICE Citizens Council. How Should NICE Assess Future Costs and Health Benefits? [Internet]. London: National Institute for Health and Care Excellence (NICE); 2011 [cited 2020 February 15]. Available from: https://www.nice.org.uk/Media/Default/Get-involved/CitizensCouncil/Reports/CCReport15Discounting.pdf.

19. Owens S, Thayer S, Frank Z, Hersh A and Caughey A. 118: Antibiotics at time of operative vaginal delivery: a cost-effectiveness analysis. American Journal of Obstetrics and Gynaecology. 2020;222(1):S92.

Table 1: Unit Cost of different healthcare resources used along the patient journey for suspected postpartum infection

\section{Treatment Component}

Unit Cost (£)

(Year)

\section{Explanation of Calculation}

Discount Rate

Present Value (£)

\section{Source}

Course of oral co-amoxiclav

4.80 (2017)

Average of 14 brands for oral suspension and tablet course of co-amoxiclav

$3.5 \%$

5.32

BNF, $2017^{8}$

Midwife home visit

19.00 (2019)

Cost of an atypically long home visit by midwife to take swabs and investigate suspected infection

$3.5 \%$

19.67

Unit costs of Health and Social care, $2019^{9}$

GP visit

28.00 (2019)

Cost of a primary care consultation (including administration staff and overheads)

$3.5 \%$

28.98

Unit costs of Health and Social Care, $2019^{9}$ 
Outpatient visit to a doctor

138.20 (2018)

Weighted average of codes 501 \& 502, total

$3.5 \%$

148.04

NHS reference cost 2017-18 10

Hospital readmission-short stay

640.30 (2018)

Weighted average of codes NZ26A \& NZ26B

$3.5 \%$

685.91

NHS reference cost 2017-18 10

Perineal wound breakdown treatment cost

$1,859.00$ (2016)

Cost of hospital admission and dressings (cost does not include district nurse visits)

$3.5 \%$

$2,133.25$

Guest, J., Fuller, G. and Vowden, P BMJ ${ }^{11}$

Endometritis Treatment

750.91 (2020)

Antibiotics cost (clindamycin and gentamicin) + Short stay Hospital treatment

750.91

D, Harding, $2017^{12}$ (provided the treatment pathway)

Clindamycin

59.00 (2020)

600mg IV Clindamycin every 6 hours for 24 hours

$-$

59.00

BNF, $2020^{13}$

Gentamicin

6.00 (2020)

80mg IV Gentamicin, 3 rounds over 24 hours 
6.00

BNF, $2020^{13}$

Co-amoxiclav 625mg PO TDS

2.41 (2020)

Perineal Wound Infection

2.41

BNF, $2020^{13}$

Amoxicillin 500mg po 8 hourly for 7 days

1.22 (2020)

UTI/other infection

1.22

NHS Prescription Services. (2020). Drug Tariff ${ }^{14}$

Paracetamol

0.73 (2020)

For perineal pain relief

\subsection{3}

BNF, $2020^{13}$

Sepsis Treatment

5686.72 (2017)

$-$

$3.5 \%$

$6,304.97$

Hex N., Retzler J., Bartlett C. and Arber M, $2017^{15}$

Hosted file

Figure 1-Decision Tree.docx available at https://authorea.com/users/337649/articles/463276prophylactic-antibiotics-with-operative-vaginal-delivery-a-cost-utility-analysis-basedon-the-anode-trial

\section{Hosted file}

Figure 2-Cost effectiveness plane.docx available at https://authorea.com/users/337649/ articles/463276-prophylactic-antibiotics-with-operative-vaginal-delivery-a-cost-utilityanalysis-based-on-the-anode-trial

\section{Hosted file}


Figure 3-Probabilistic analysis.docx available at https://authorea.com/users/337649/articles/ 463276-prophylactic-antibiotics-with-operative-vaginal-delivery-a-cost-utility-analysisbased-on-the-anode-trial 